\title{
Description of four new species of the subgenus Tachycines (Gymnaeta) Adelung, 1902 (Orthoptera: Rhaphidophoridae) from caves in China and additional notes on some previously known species
}

\author{
Qi-Di ZHU ${ }^{\circledR}{ }^{1}$ \& Fu-Ming SHI ${ }^{\circledR 2, *}$ \\ ${ }^{1,2}$ College of Life Science, Institute of Life Science and Green Development, Hebei University, \\ Baoding 071002, P.R. China. \\ *Corresponding author: shif_m@126.com \\ 1Email: qidizhu0323@163.com \\ ${ }^{1}$ urn:lsid:zoobank.org:author:7796C4B5-5DC2-42BB-A671-18BE36E68C48 \\ ${ }^{2}$ urn:lsid:zoobank.org:author:9E53A7F0-4FEB-4AED-8E3C-BD1AB497154D
}

\begin{abstract}
In this paper, four new cave-dwelling species of the subgenus Tachycines (Gymnaeta) from Guangxi, Hunan and Jiangxi are described, i.e., Tachycines (Gymnaeta) quadratus sp. nov., Tachycines (Gymnaeta) shiziensis sp. nov., Tachycines (Gymnaeta) sparsispinus sp. nov. and Tachycines (Gymnaeta) papilious sp. nov. The female of Tachycines (Gymnaeta) liboensis Zhu, Chen \& Shi, 2020 is recorded for the first time. Moreover, images illustrating the morphology of the four new and three previously described species are provided.
\end{abstract}

Keywords. Rhaphidophoridae, Tachycines (Gymnaeta), new species, caves, China.

Zhu Q.-D. \& Shi F.-M. 2021. Description of four new species of the subgenus Tachycines (Gymnaeta) Adelung, 1902 (Orthoptera: Rhaphidophoridae) from caves in China and additional notes on some previously known species. European Journal of Taxonomy 764: 1-17. https://doi.org/10.5852/ejt.2021.764.1465

\section{Introduction}

In recent years, the diversity of cave species in China has attracted widespread attention, especially in studies on spiders, pseudoscorpions, beetles and millipedes (Tian et al. 2017; Jiang et al. 2018; Liu \& Wynne 2019; Feng Z.G. et al. 2020; Gao et al. 2020; Zhao et al. 2020; Ma et al. 2020). The study of cave crickets from karstic areas in Southern China began with several biospeleological expeditions in the 1990s, organized by Chinese, French, Italian and Slovenian teams. In total, 11 species (subspecies) were discovered and described from Guizhou, Guangxi, Hunan and Hubei (Gorochov et al. 2006; Rampini et al. 2008). Jiao et al. (2008) recorded one species, Diestrammena (G.) caverna Jiao, Niu, Liu, Lei \& Bi, 2008, from Yinshui Cave in Hubei. After that, cave crickets did not attract scholars' attention for a decade. Since 2019, an increasing number of species has been reported (Feng X.L. et al. 2019, 2020a, 2020b; Zhou \& Yang 2020; Zhu et al. 2020). Up to now, the subgenus Tachycines (Gymnaeta) contains 25 cave-dwelling species (Gorochov et al. 2006; Rampini et al. 2008; Jiao et al. 2008; Qin et al. 2019; Feng X.L. et al. 2019, 2020a, 2020b; Zhou \& Yang 2020; Zhu et al. 2020; Cigliano et al. 2020). 
This paper describes four new species from caves in Guangxi, Hunan and Jiangxi. Images showing the morphology of the four new and three previously described species are provided.

\section{Material and methods}

Specimens were preserved in $75 \%$ ethanol and measured by Vernier caliper. Morphological structures were examined using a Leica M205A stereo microscope and a Leica DFC450 digital imaging system was used to acquire morphological images. The photos were edited with Adobe Photoshop CC 2018. Type specimens are kept at the Museum of Hebei University, Baoding, China (HBU).

The following conventions were adopted for the specimen measurements: body length from apex of fastigium verticis to posterior margin of the last abdominal tergite; pronotum length from anterior margin of pronotum to posterior margin along midline; fore femur length from base of fore femur to apex of genicular lobe; hind femur length from base of hind femur to apex of genicular lobe; hind tibia length from base of hind tibia to apex; hind basitarsus length from base of hind basitarsus to apex; ovipositor length from base of subgenital plate to apex of ovipositor.

\section{Results}

Class Insecta Linnaeus, 1758

Order Orthoptera Latreille, 1810

Superfamily Rhaphidophoroidea Walker, 1869

Family Rhaphidophoridae Walker, 1869

Subfamily Aemodogryllinae Jacobson, 1905

Tribe Aemodogryllini Jacobson, 1905

Genus Tachycines Adelung, 1902

Subgenus Tachycines (Gymnaeta) Adelung, 1902

Tachycines (Gymnaeta) quadratus sp. nov. urn:1sid:zoobank.org:act:A76373AC-5FF4-4ED9-8496-A4180B9D1456

Figs $1,8 \mathrm{~A}-\mathrm{B}$

\section{Diagnosis}

The new species is similar to Tachycines (Gymnaeta) sonlaensis Gorochov, 1990 with regard to the dorsal sclerite of the male genitalia, but differs from the latter by: projection of fastigium verticis is fully reduced; hind tibia has a lower number of dorsal spines (only 11-13 inner spines and 10-14 outer spines instead of 30-40 spines in $T$. (G.) sonlaensis); interno-dorsal spine is distinctly shorter than hind basitarsus instead of nearly equal in length to hind basitarsus in $T$. $(G$.) sonlaensis.

\section{Etymology}

The name of the new species derives from the Latin word 'quadratus' referring to the dorsal sclerite of the male genitalia quadrate being quadrangular.

\section{Material examined}

Holotype

CHINA • đ̃; Hunan Prov., Yongzhou City, Lanshan County, Dibao Cave; 2 Oct. 2011; Ming-Yi Tian leg.; HBU.

\section{Paratypes}

CHINA 1 ภ, 1 ; ; same collection data as for holotype; HBU. 


\section{Description}

BoDy. Medium-sized.

HEAD. Fastigium verticis fully reduced. Eyes slightly degenerated, oval; medium ocellus oval, located between antennal sockets. Apical segment of maxillary palpus distinctly longer than subapical one, apical area inflated, globular.

THORAX. Pronotum long, anterior margin straight, posterior margin arcuate; lateral lobes longer than high, ventral margin arc-shaped. Mesonotum and metanotum short, posterior margin of mesonotum arcuate, posterior margin of metanotum straight.
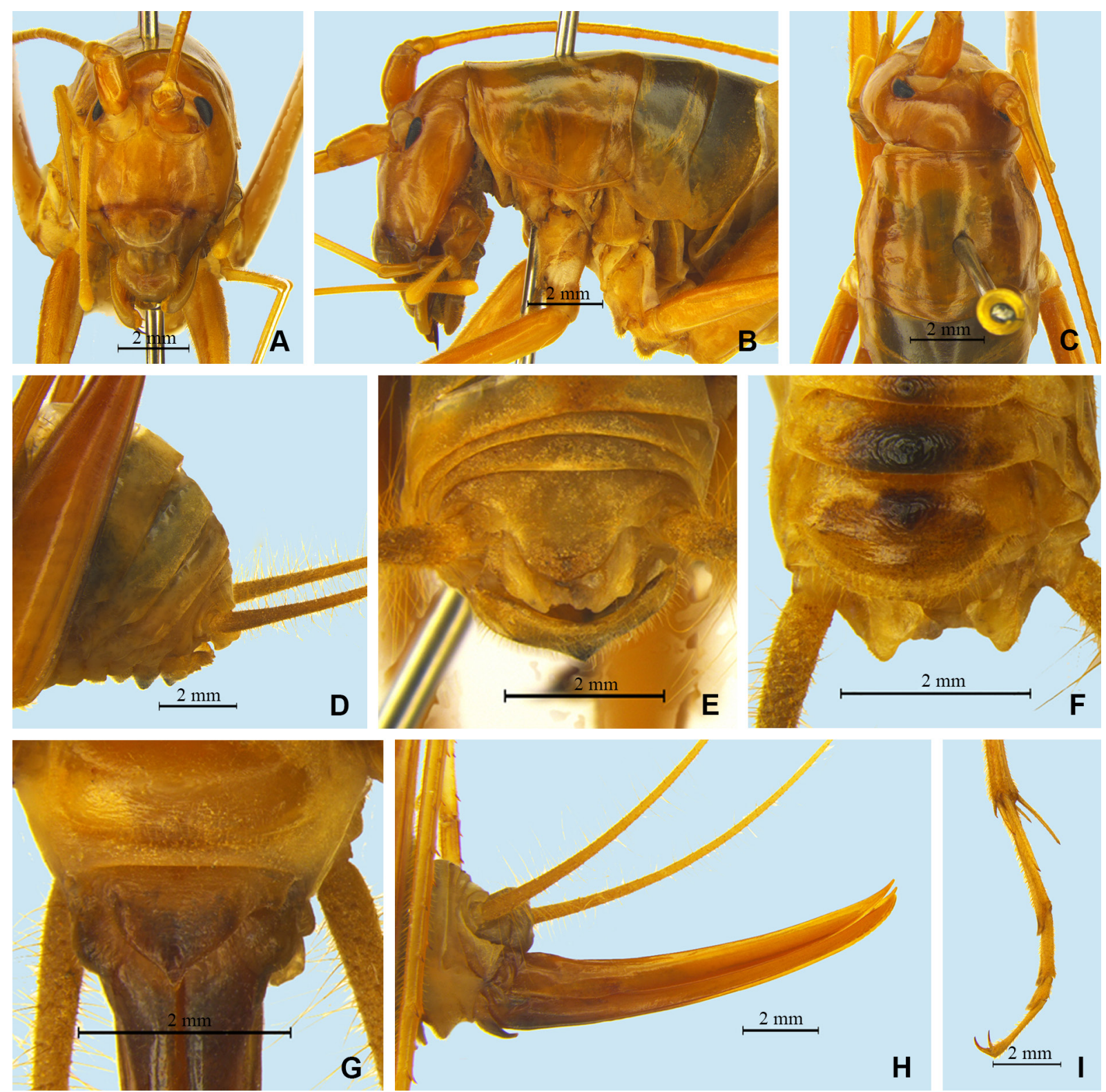

Fig. 1. Tachycines (Gymnaeta) quadratus sp. nov. A-F, I. Holotype, ồ (HBU). A-C. Head and pronotum. A. Frontal view. B. Lateral view. C. Dorsal view. D-F. Apex of abdomen. D. Lateral view. E. Dorsal view. F. Ventral view. I. Hind tarsus in lateral view. - G-H. Paratype, $q$ (HBU). G. Subgenital plate. H. Ovipositor in lateral view. 
Legs. Fore coxa with one small spine; femur unarmed on ventral surface, internal genicular lobe with one small spine, external genicular lobe with one long spine; tibia with one inner spine and one to two outer spines on ventral surface, apex with one pair of ventral spines, between paired ventral spines with one small spine. Mid femur unarmed on ventral surface, internal and external genicular lobes with one long spine each; tibia with only one outer spine on ventral surface, apex with one inner spine on dorsal surface and one pair of ventral spines, between paired ventral spines with one small spine. Hind femur unarmed on ventral surface, internal and external genicular lobes unarmed; tibia with 11-13 inner spines and 10-14 outer spines on dorsal surface, arranged sparsely, with one pair of dorsal spines in subapical area, and at apex with one pair of dorsal spines and two pairs of ventral spines, interno-dorsal spine distinctly shorter than hind basitarsus. Hind basitarsus unarmed on dorsal surface, ventral surface with bristles.

MALE ABdomen. Posterior margins of all abdominal tergites relatively straight, abdominal sterna with conical tubercles. Epiproct tongue-shaped, paraproct simple, nearly triangular in lateral view. Cercus slender, conical, apex acute. Genitalia with six membranous lobes, apical area of dorso-median lobe with one pair of small lobes, lateral sclerite indistinct, dorso-lateral lobes nearly equal in length to dorsomedian lobe, ventro-lateral lobes nearly equal in length to dorso-lateral lobes, ventro-median lobe short; dorsal sclerite quadrate. Subgenital plate quadrangular, transverse and broad, middle area with conical tubercle, posterior margin straight.

Female abdomen. Ovipositor longer than half the length of hind femur, slightly curved upward, dorsal margin smooth, apical area of ventral margin denticulate. Subgenital plate nearly triangular, both sides of basal area with one triangulate lobe, apex acute.

Coloration. Body yellowish-brown. Eyes black, conical tubercles of abdominal sterna black.

\section{Measurements (mm)}

Body length: $\widehat{\jmath}: 15.30-15.70$, $q$ : 16.34; length of pronotum: $\widehat{\delta}: 4.44-4.60$, $q: 4.18$; length of fore femur: ${ }^{\lambda}: 11.94-12.56$, o $: 11.90$; length of hind femur: $\delta^{\lambda}: 20.10-20.20$, o $: 19.04$; length of hind tibia:

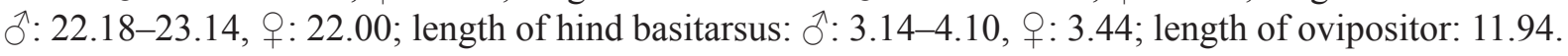

\section{Distribution}

China (Hunan).

Tachycines (Gymnaeta) shiziensis sp. nov. urn:lsid:zoobank.org:act:5C1282B7-963A-4856-B626-1CBD08E99306

Figs 2, 8C-D

\section{Diagnosis}

The new species is similar to other congeneric species in appearance, but it can easily be distinguished by the shape of the dorsal sclerite of the male genitalia, the dorsal sclerite being trapezoidal, the basal part narrow, with one deep concavity, and the posterior margin being broad and slightly concave.

\section{Etymology}

The name of the new species refers to the type locality.

\section{Material examined}

Holotype

CHINA • '̇; Jiangxi Prov., Ji'an City, Wan'an County, Baoshan Village, Shiziyan; 16 Oct. 2013; MingYi Tian, Wei-Xin Liu, Hao-Min Yin and Xin-Hui Wang leg.; HBU. 


\section{Description}

Male

BoDy. Slightly smaller than in other congeneric species.

HeAd. Fastigium verticis degenerated, indistinct. Eyes oval, protruding forward. Apical segment of maxillary palpus distinctly longer than subapical one, apex inflated, globular.

Thorax. Pronotum long, anterior margin straight, posterior margin arcuate; lateral lobes longer than high, ventral margin arc-shaped. Mesonotum and metanotum short, posterior margin of mesonotum arcuate, posterior margin of metanotum straight.

LEGS. Fore coxa with one small spine; femur unarmed on ventral surface, internal genicular lobe unarmed, external genicular lobe with one long spine; tibia with only two outer spines on ventral surface, apex with one pair of ventral spines, between paired ventral spines with one small spine. Mid femur unarmed on ventral surface, internal and external genicular lobes with one long spine each; tibia unarmed on ventral surface, apex with one pair of ventral spines, between paired ventral spines with one small spine. Hind femur unarmed on ventral surface, internal and external genicular lobes unarmed; tibia with 23-24 inner spines and 21-24 outer spines on dorsal surface, with one pair of dorsal spines in subapical area, and at apex with one pair of dorsal spines and two pairs of ventral spines, interno-dorsal spine slightly shorter than hind basitarsus. Hind basitarsus with one apical spine on dorsal surface, ventral surface with bristles.

MALE ABDOMEn. Posterior margins of all abdominal tergites relatively straight, abdominal sterna without obvious conical tubercles. Epiproct tongue-shaped, paraproct simple, nearly triangular in lateral view. Cercus extremely long, conical, apex acute. Genitalia with six membranous lobes, apical area of dorso-
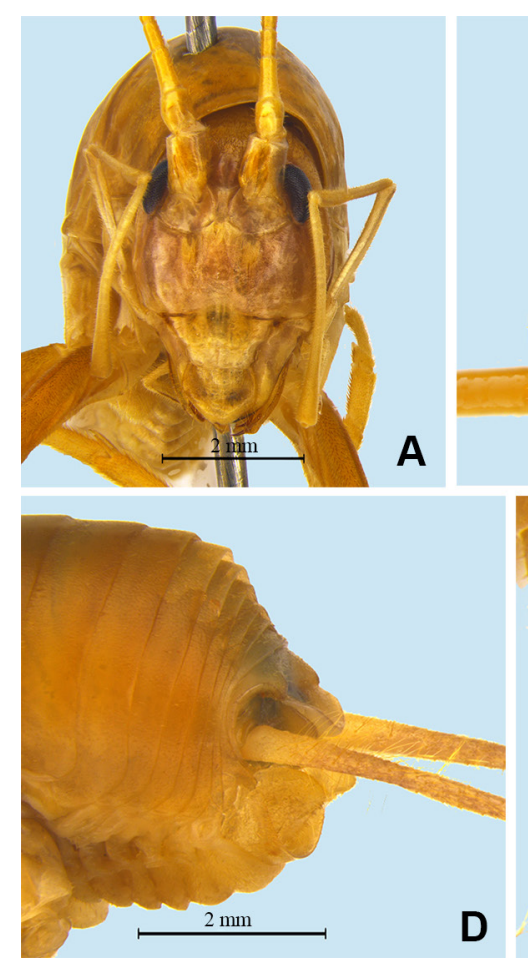
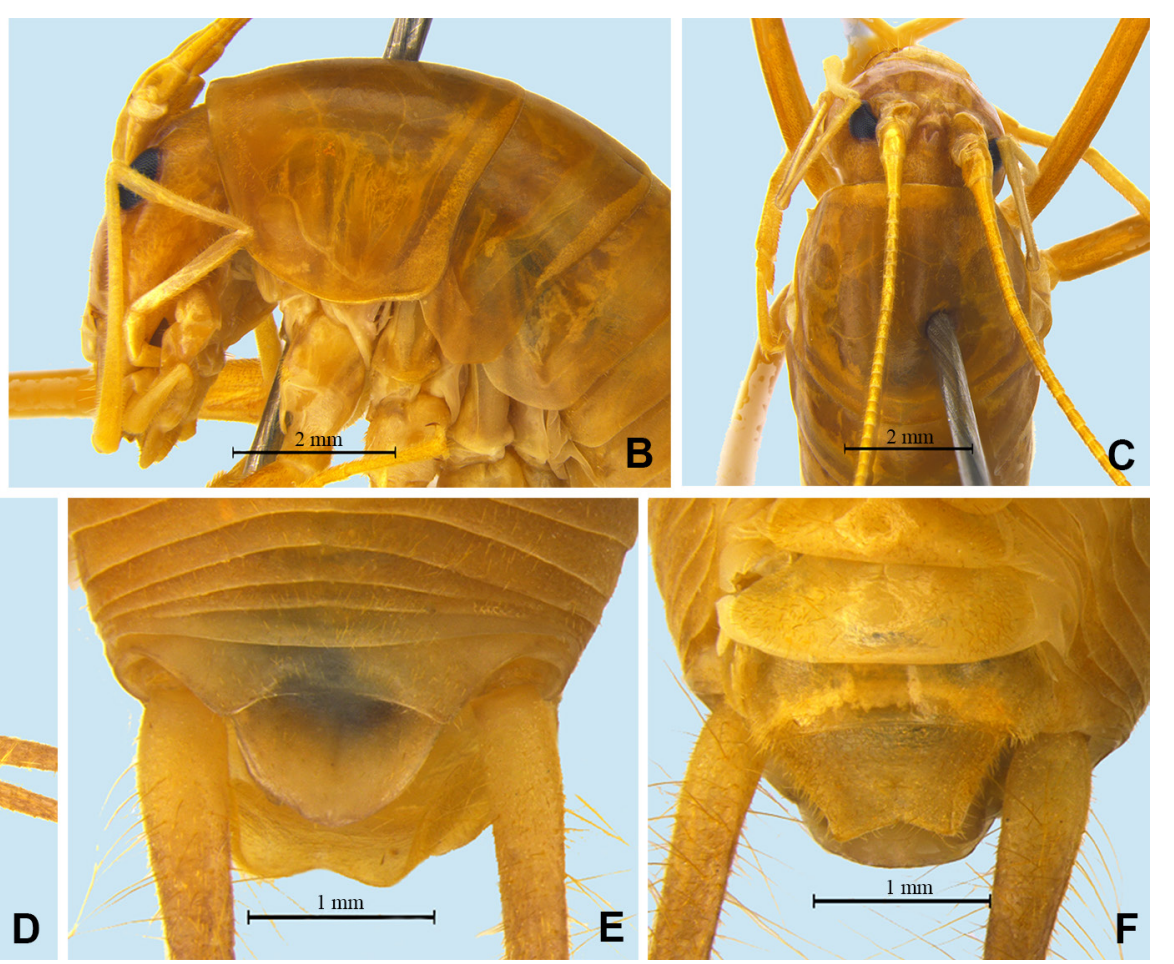

Fig. 2. Tachycines (Gymnaeta) shiziensis sp. nov. A-F. Holotype, $\widehat{\jmath}$ (HBU). A-C. Head and pronotum. A. Frontal view. B. Lateral view. C. Dorsal view. D-F. Apex of abdomen. D. Lateral view. E. Dorsal view. F. Ventral view. 
median lobe with one pair of small lobes, lateral sclerite slender, reaching subapex of dorso-median lobe, dorso-lateral lobes slightly longer than dorso-median lobe, ventro-lateral lobes much shorter than dorso-lateral lobes, ventro-median lobe short; dorsal sclerite trapezoidal, basal part narrow, with one deep concavity, posterior margin broad and slightly concave. Subgenital plate quadrangular, posterior margin slightly concave.

Coloration. Body uniformly yellowish-brown. Eyes black.

Female

Unknown.

\section{Measurements (mm)}

Body length: $\delta: 9.90$; length of pronotum: 0 : 3.54 ; length of fore femur: $0: 7.74$; length of hind femur: t: 13.80 ; length of hind tibia: $\delta^{\lambda}: 14.20$; length of hind basitarsus: $\delta^{\lambda}: 2.80$.

\section{Distribution}

China (Jiangxi).

Tachycines (Gymnaeta) sparsispinus sp. nov. urn:Isid:zoobank.org:act:BF2EF6CF-707A-41F6-A858-C6CECC4625BE

Figs 3, 8E-F

\section{Diagnosis}

The new species is similar to Tachycines (Gymnaeta) bifolius Zhu, Chen \& Shi, 2020 and Tachycines (Gymnaeta) fallax (Zhang \& Liu, 2009), but differs from T. (G.) bifolius by: eyes degenerated, taenioid; hind tibia with six to eight inner and outer spines each on dorsal surface; apical area of dorso-median lobe of male genitalia with one pair of small lobes; female subgenital plate is nearly triangular. The new species differs from $T$. (G.) fallax by: fastigium verticis completely reduced, eyes degenerated, hind tibia with six to eight inner and outer spines each on dorsal surface.

\section{Etymology}

The name of the new species is formed from the Latin words 'sparsus' ('scattered') and 'spina' ('spine'), referring to the low number of dorsal spines on the hind tibia.

\section{Material examined}

\section{Holotype}

CHINA - ô; Guangxi Zhuang Autonomous Region, Baise City, Tianlin County, Langping Village, Shizikou Cave; 13 Jun. 2014; Ming-Yi Tian, Wei-Xin Liu, Hao-Min Yin \& Xiao-Zhu Luo leg.; HBU.

\section{Paratypes}

CHINA $\bullet 2 \widehat{\partial} \widehat{\partial}, 1$; ; same collection data as for holotype; HBU.

\section{Description}

Body. Medium-sized.

HEAD. Fastigium verticis fully reduced. Eyes degenerated, taenioid. Apical segment of maxillary palpus distinctly longer than subapical one, apex inflated, globular.

Thorax. Pronotum long, anterior margin straight, posterior margin arcuate; lateral lobes longer than high, ventral margin arc-shaped. Mesonotum and metanotum short, posterior margins straight. 
LEGS. Fore coxa with one small spine; femur unarmed on ventral surface, internal genicular lobe unarmed, external genicular lobe with one long spine; tibia with one inner spine and two outer spines on ventral surface, apex with one pair of ventral spines, between paired ventral spines with one small spine. Mid femur unarmed on ventral surface, internal and external genicular lobes with one long spine each; tibia with one inner spine and one to two outer spines on ventral surface, apex with one pair of dorsal spines and one pair of ventral spines, between paired ventral spines with one small spine. Hind femur unarmed on ventral surface, internal and external genicular lobes unarmed; tibia with six to eight inner and outer spines on dorsal surface each, subapical area unarmed, and at apex with one pair of dorsal spines and two pairs of ventral spines, interno-dorsal spine distinctly shorter than hind basitarsus. Hind basitarsus unarmed on dorsal surface, ventral surface with bristles.
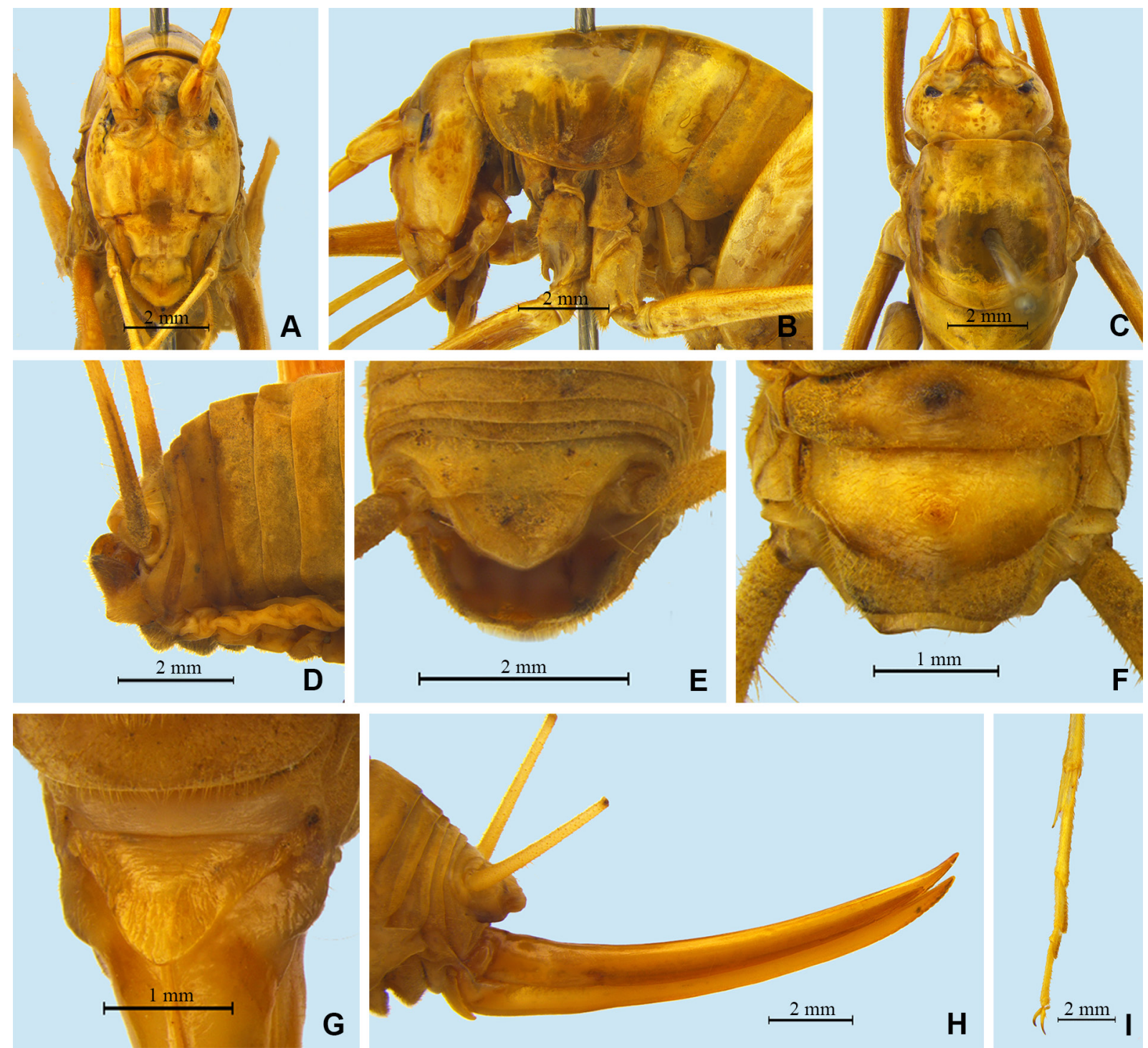

Fig. 3. Tachycines (Gymnaeta) sparsispinus sp. nov. A-F, I. Paratype, § (HBU). A-C. Head and pronotum. A. Frontal view. B. Lateral view. C. Dorsal view. D-F. Apex of abdomen. D. Lateral view. E. Dorsal view. F. Ventral view. I. Hind tarsus in lateral view. - G-H. Paratype, $q$ (HBU). G. Subgenital plate. H. Ovipositor in lateral view. 
MALE ABDOMEn. Posterior margins of all abdominal tergites relatively straight, abdominal sterna without obviously conical tubercles. Epiproct tongue-shaped, paraproct simple, nearly triangular in lateral view. Cercus slender, conical, apex acute. Genitalia with six membranous lobes, apical area of dorso-median lobe with one pair of small lobes, lateral sclerite short, basal area broad, gradually narrowing to apex, dorso-lateral lobes nearly equal in length to dorso-median lobe, ventro-lateral lobes nearly equal in length to dorso-lateral lobes, ventro-median lobe short; basal margin of dorsal sclerite narrow, truncate, posterior margin with one broad concavity. Subgenital plate quadrangular, posterior margin relatively straight.

FEMALE ABDOMEN. Ovipositor longer than half the length of hind femur, slightly curved upward, dorsal margin smooth, apical area of ventral margin denticulate. Subgenital plate nearly triangular, both sides of basal area with one triangulate lobe, apex blunt.

Coloration. Body uniformly yellowish-brown. Eyes black.

\section{Measurements (mm)}

Body length: $\delta^{7}: 14.16-14.56$, $q$ : 17.76; length of pronotum: $\delta: 3.80-4.42$, + : 4.0; length of fore femur: $\delta^{1}: 11.08-11.80$, , $: 11.50$; length of hind femur: $\delta^{1}: 18.50-18.54$, $q: 17.90$; length of hind tibia:

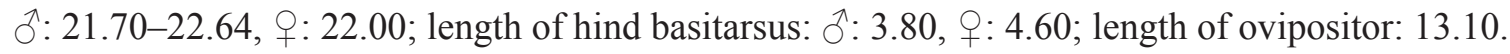

\section{Distribution}

China (Guangxi).

\section{Tachycines (Gymnaeta) papilious sp. nov. urn:lsid:zoobank.org:act:E887A212-5447-456A-88EB-CDA69C7884C2}

Figs 4, 8G-H

\section{Diagnosis}

The new species is similar to other congeneric species in appearance, but it can easily be distinguished by the dorsal sclerite of the male genitalia being shaped like a butterfly with open wings, the basal area of the dorso-median lobe forming a protuberance, and the hind tibia being provided with 104 inner spines and 114 outer spines on the dorsal surface.

\section{Etymology}

The name of the new species derives from the Latin word 'papilio' ('butterfly'), referring to the dorsal sclerite of the male genitalia being shaped like a butterfly with open wings.

\section{Material examined}

\section{Holotype}

CHINA • đ’; Hunan Prov., Chenzhou City, Wanhuayan; 23 Aug. 2015; Xin-Hui Wang, Sun-Bin Huang, Ming-Ruo Tang and Ping-Jing Yang leg.; HBU.

\section{Description}

\section{Male}

Body. Medium-sized.

HEAD. Fastigium verticis with two conical tubercles, apices obtusely rounded, pointing forward. Eyes oval, protruding forward; median ocellus oval, located between antennal sockets; lateral ocelli nearly 
circular, situated on lateral margin of basal area of fastigium verticis. Apical segment of maxillary palpus distinctly longer than subapical one, apex inflated, globular.

THORAX. Pronotum long, anterior margin straight, posterior margin arcuate; lateral lobes longer than high, ventral margin arc-shaped. Mesonotum and metanotum short, posterior margin of mesonotum arcuate, posterior margin of metanotum straight.

LEGS. Fore coxa with one small spine; femur unarmed on ventral surface, internal genicular lobe with one small spine, external genicular lobe with one long spine; tibia with one inner spine and two outer spines on ventral surface, apex with one outer spine on dorsal surface and one pair of ventral spines, between paired ventral spines with one small spine. Mid femur unarmed on ventral surface, internal and external genicular lobes with one long spine each; tibia with one inner spine and one outer spine on ventral surface, apex with one pair of dorsal spines and one pair of ventral spines, between paired ventral spines with one small spine. Hind femur unarmed on ventral surface, internal genicular lobe with one spine, external genicular lobe unarmed; tibia with 104 inner spines and 114 outer spines on dorsal surface, with one pair of dorsal spines in subapical area, and at apex with one pair of dorsal spines and two pairs of ventral spines, interno-dorsal spine markedly shorter than hind basitarsus. Hind basitarsus with one apical spine on dorsal surface, ventral surface without bristles.

MALE ABDOMEN. Posterior margins of all abdominal tergites relatively straight, abdominal sterna with distinct conical tubercles. Epiproct semicircular, paraproct simple, nearly triangular in lateral view. Cercus extremely long, conical, apex acute. Genitalia with six membranous lobes, basal area of dorsomedian lobe forming a protuberance, apical area with one pair of small lobes, lateral sclerite narrow, crescent shaped, dorso-lateral lobes slightly longer than dorso-median lobe, ventro-lateral lobes shorter
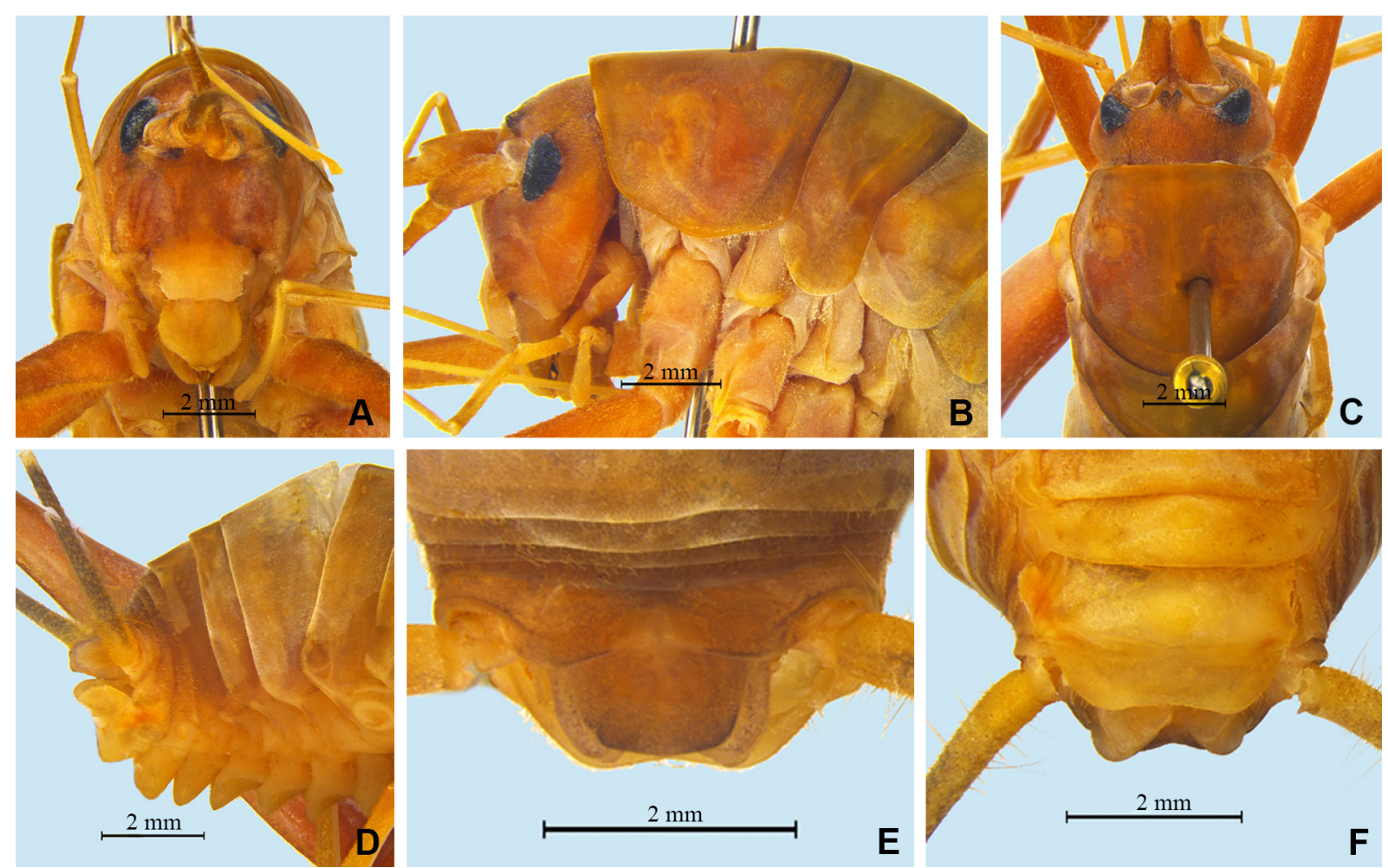

Fig. 4. Tachycines (Gymnaeta) papilious sp. nov. A-F. Holotype, ふ̋ (HBU). A-C. Head and pronotum. A. Frontal view. B. Lateral view. C. Dorsal view. D-F. Apex of abdomen. D. Lateral view. E. Dorsal view. F. Ventral view. 
than dorso-lateral lobes, ventro-median lobe short; dorsal sclerite shaped like a butterfly with open wings. Subgenital plate quadrangular, posterior margin slightly concave.

Coloration. Body uniformly yellowish-brown. Eyes black.

\section{Female}

Unknown.

Measurements (mm)

Body length: $\delta$ : 16.54; length of pronotum: $\delta: 5.10$; length of fore femur: $\delta: 13.58$; length of hind femur: $\delta^{\top}: 26.28$; length of hind tibia: $\widehat{\delta}: 29.40$; length of hind basitarsus: $\delta^{\Uparrow}: 5.04$.

\section{Distribution}

China (Hunan).

Tachycines (Gymnaeta) liboensis Zhu, Chen \& Shi, 2020

Fig. 5

Tachycines (Gymnaeta) liboensis Zhu, Chen \& Shi, 2020: 73.

\section{Material examined}

\section{Holotype}

CHINA - Ō; Guizhou Prov., Libo County, Dongtang Village, Banzhai Hamlet, Maan Cave; 25 Oct. 2009; Hui-Ming Chen leg.; HBU.

\section{Additional material}

CHINA $\bullet 1 \hat{\jmath}$, Guangxi Zhuang Autonomous Region, Huanjiang County, Chuanshan Village, Xiarong Hamlet, Shuiyuan Cave; 4 Oct. 2016; Ming-Yi Tian, Ju-Jian Chen, Xin-Hui Wang, Ming-Ruo Tang, Dian-Mei Wang, Zhuang-Hui Qin and Rui-Chong Wang leg.; HBU • 1 \%; Guangxi Zhuang Autonomous Region, Huanjiang County, Chuanshan Village, Xiarong Hamlet, The Second Cave; 6 Oct. 2016; MingYi Tian, Ju-Jian Chen, Xin-Hui Wang, Ming-Ruo Tang, Dian-Mei Wang, Zhuang-Hui Qin and RuiChong Wang leg.; HBU.

\section{Descriptive notes of the female}

Ovipositor nearly equal in length to half the length of hind femur, slightly curved upward, dorsal margin smooth, apical area of ventral margin denticulate. Subgenital plate nearly triangular, both sides of basal area with one triangular lobe, apex blunt.

\section{Measurements (mm)}

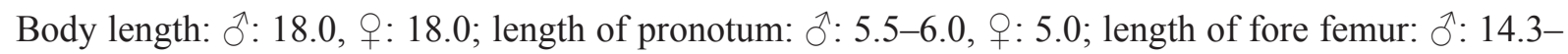
15.0,, : 12.2 ; length of hind femur: $\delta^{\lambda}: 26.5-27.0$,, : 23.0 ; length of hind tibia: $\delta^{\lambda}: 30.0-31.0$, q $: 27.0$; length of hind basitarsus: 0 : $6.0,+$, $: 5.0$; length of ovipositor: 11.8 .

\section{Distribution}

China (Guangxi, Guizhou).

\section{Remarks}

The female of this species is recorded and described here for the first time. 

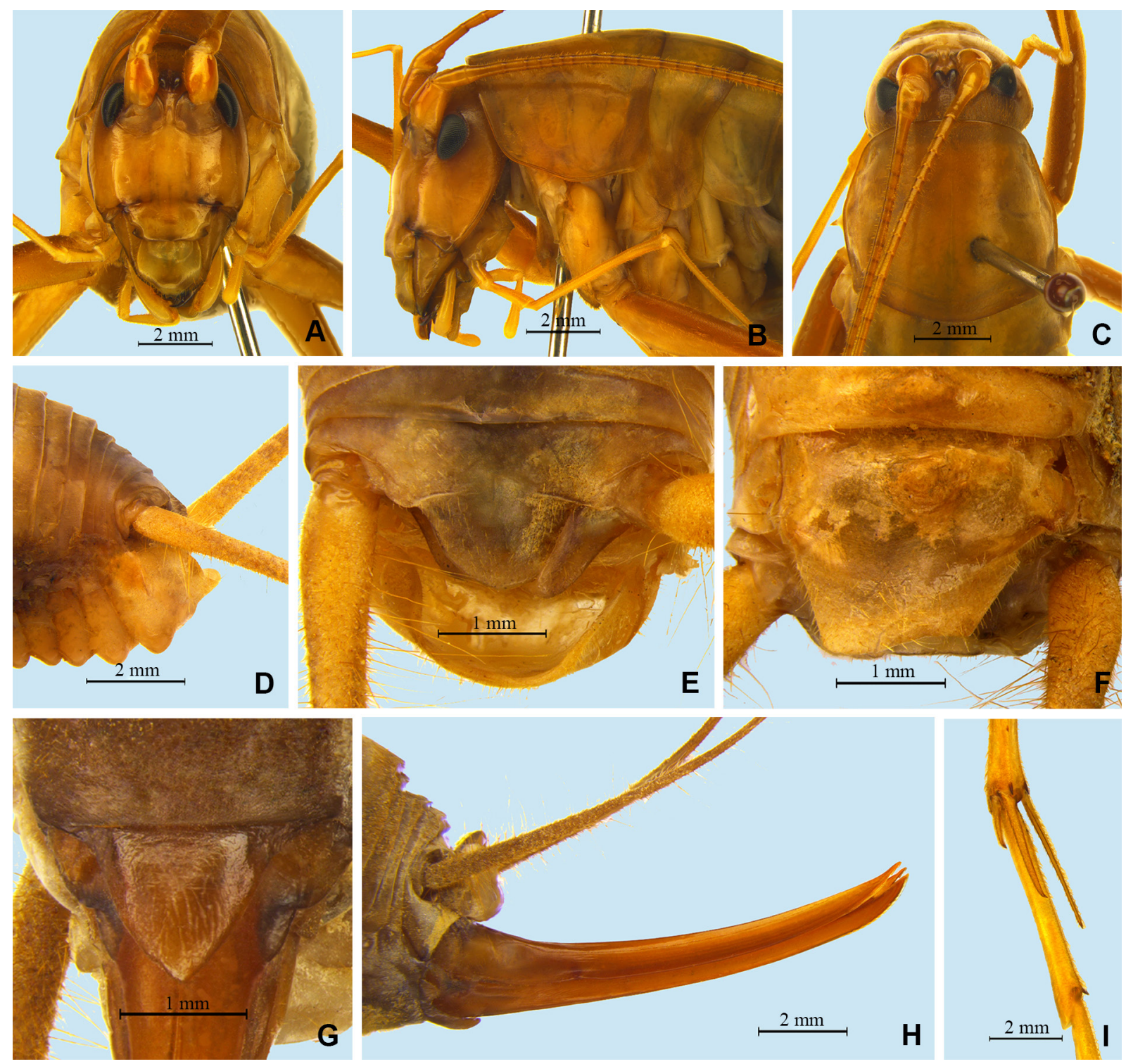

Fig. 5. Tachycines (Gymnaeta) liboensis Zhu, Chen \& Shi, 2020. A-F, I. ڤ̂. A-C. Head and pronotum. A. Frontal view. B. Lateral view. C. Dorsal view. D-F. Apex of abdomen. D. Lateral view. E. Dorsal view. F. Ventral view. I. Hind basitarsus in lateral view. - G-H. ․ . G. Subgenital plate. H. Ovipositor in lateral view.

Tachycines (Gymnaeta) latellai (Rampini \& Di Russo, 2008)

Figs 6, 8I-J

Diestrammena (Gymnaeta) latellai Rampini, Di Russo \& Cobolli, 2008: 32.

Tachycines (Gymnaeta) latellai - Qin, Wang, Liu \& Li 2018: 452.

\section{Material examined}

CHINA • 1 Ĵ, 1 क; Guizhou Prov., Bijie City, Zhijin County, Chengguan Village, Dongshan Hamlet,

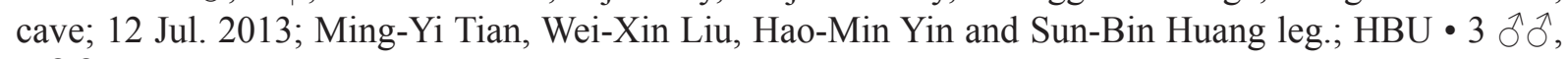
7 우; Guizhou Prov., Bijie City, Zhijin County, Zhijin Cave; 14 Aug. 2020; Wei Yang leg.; HBU. 


\section{Distribution}

China (Guizhou).
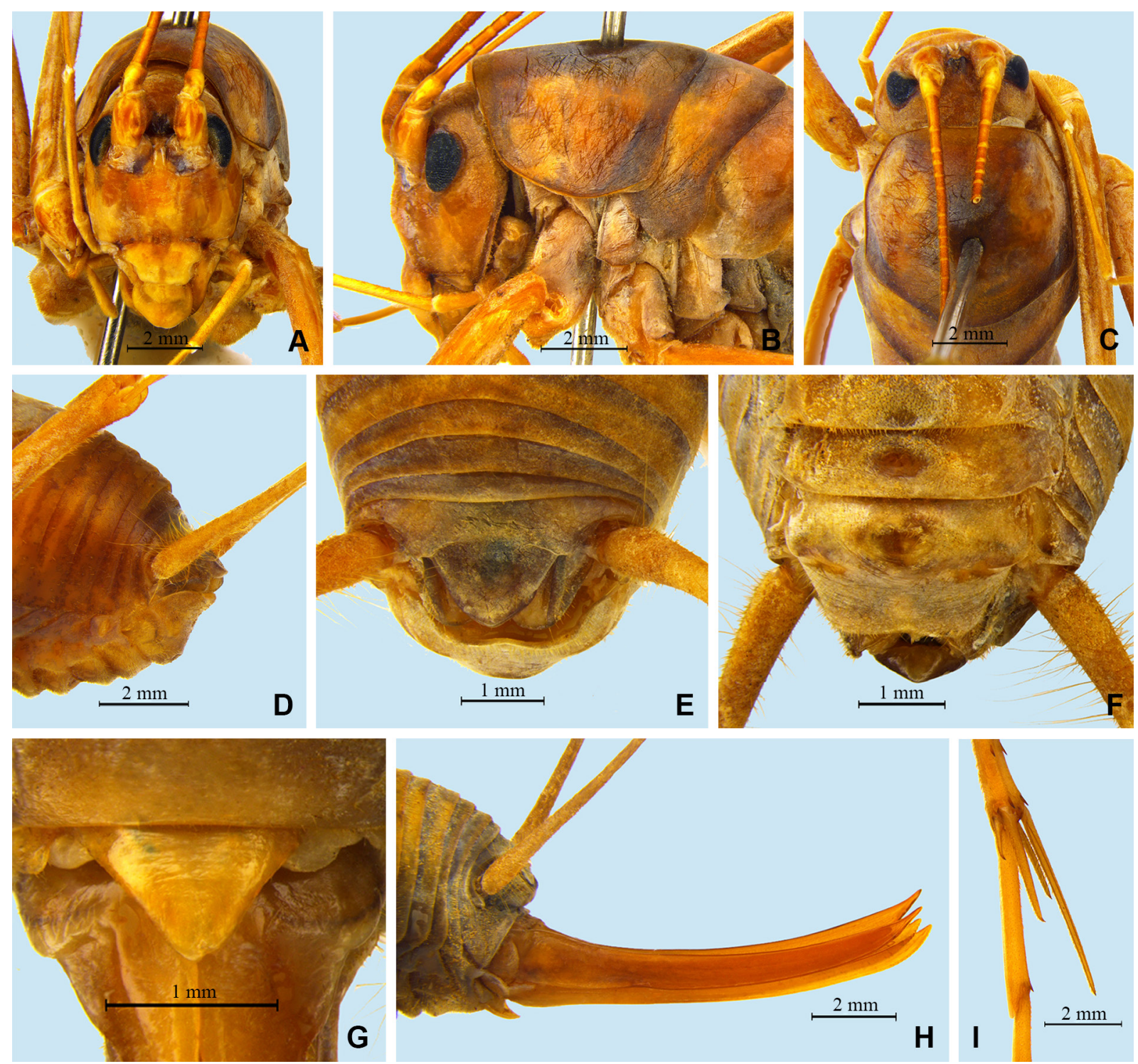

Fig. 6. Tachycines (Gymnaeta) latellai (Rampini \& Di Russo, 2008). A-F, I. §. A-C. Head and pronotum. A. Frontal view. B. Lateral view. C. Dorsal view. - D-F. Apex of abdomen. D. Lateral view. E. Dorsal view. F. Ventral view. I. Hind basitarsus in lateral view. - G-H. q. G. Subgenital plate. H. Ovipositor in lateral view.

Tachycines (Gymnaeta) omninocaecus (Gorochov, Rampini \& Di Russo, 2006) Figs $7,8 \mathrm{~K}-\mathrm{L}$

Diestrammena (Gymnaeta) omniocaeca Gorochov, Rampini \& Di Russo, 2006: 358.

Tachycines (Gymnaeta) omninocaecus - Qin, Wang, Liu \& Li 2018: 452. 


\section{Material examined}

CHINA • 1 ふै, 1 q; Hunan Prov., Longshan County, Huoyan Village, Panlong Cave; 3 Jul. 2014; MingYi Tian, Wei-Xin Liu, Hao-Min Yin, Sun-Bin Huang and Xin-Hui Wang leg.; HBU.

\section{Distribution}

China (Hunan).
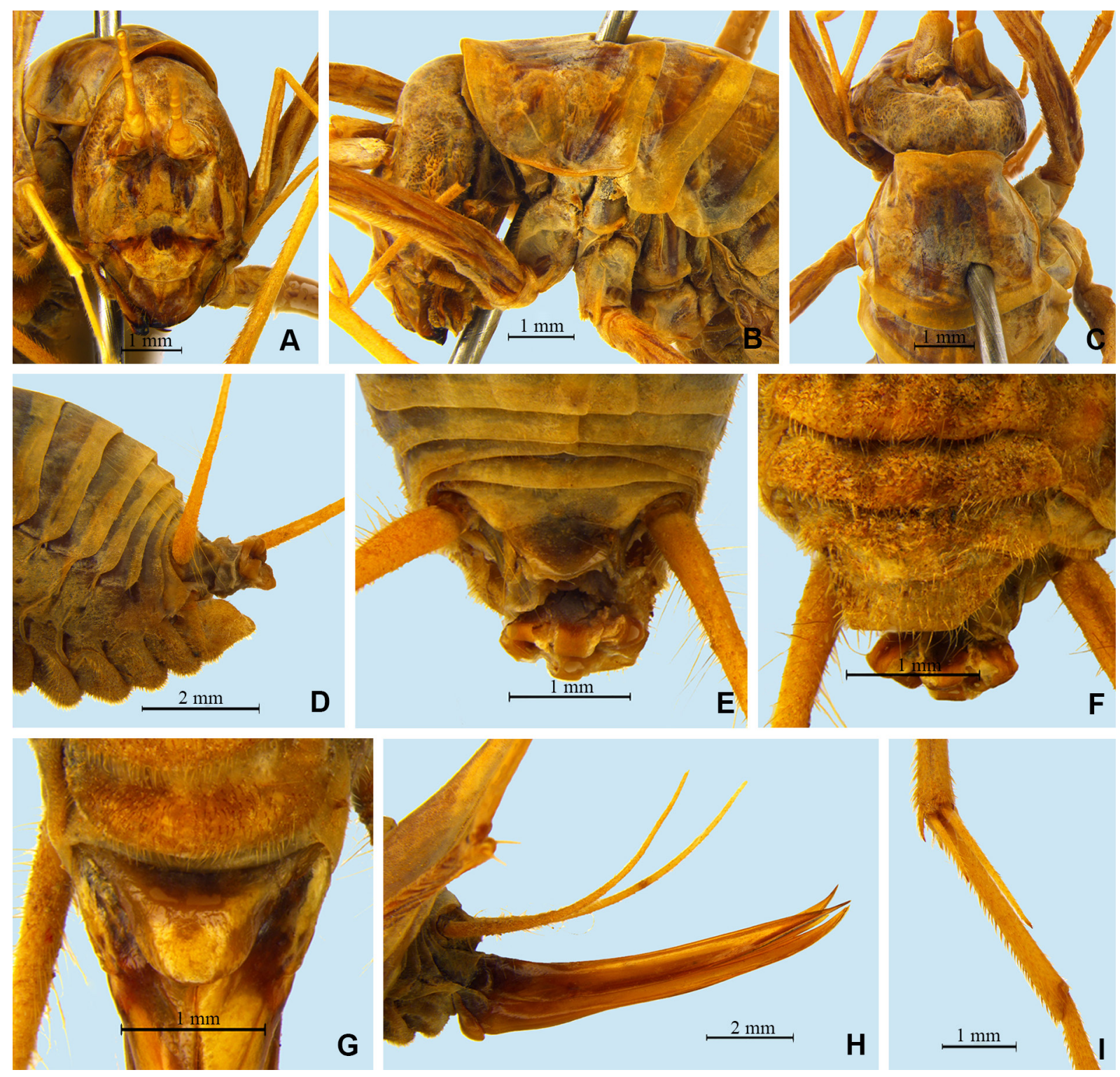

Fig. 7. Tachycines (Gymnaeta) omninocaecus (Gorochov, Rampini \& Di Russo, 2006). A-F, I. ठ̊. A-C. Head and pronotum. A. Frontal view. B. Lateral view. C. Dorsal view. D-F. Apex of abdomen. D. Lateral view. E. Dorsal view. F. Ventral view. I. Hind basitarsus in lateral view. - G-H. ㅇ. G. Subgenital plate. H. Ovipositor in lateral view. 

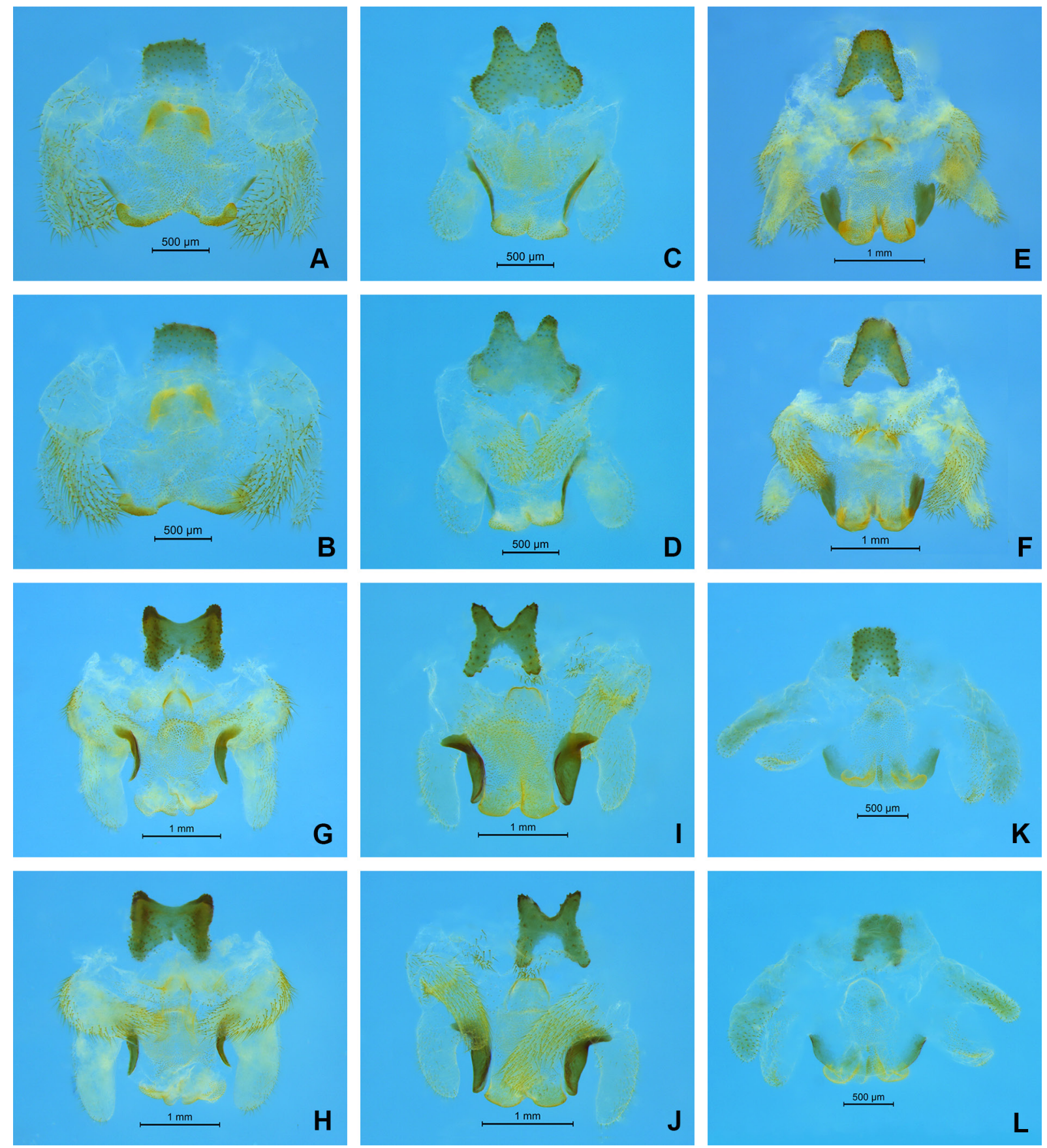

Fig. 8. Male genitalia. A, C, E, G, I, K. Dorsal view. B, D, F, H, J, L. Ventral view. A-B. Tachycines (Gymnaeta) quadratus sp. nov., holotype, ô (HBU). C-D. Tachycines (Gymnaeta) shiziensis sp. nov., holotype, ô (HBU). E-F. Tachycines (Gymnaeta) sparsispinus sp. nov., holotype, đิ (HBU). G-H. Tachycines (Gymnaeta) papilious sp. nov., holotype, ô (HBU). I-J. Tachycines (Gymnaeta) latellai (Rampini \& Di Russo, 2008), §̂. K-L. Tachycines (Gymnaeta) omninocaecus (Gorochov, Rampini \& Di Russo, 2006), $\widehat{\jmath}$. 


\section{Discussion}

Gymnaeta was erected as a genus by Adelung (1902). Karny (1934) considered it as a subgenus of Tachycines. Gorochov \& Storozhenko (1992) compared the genus Diestrammena with the genus Tachycines and thought that the structure of their male genitalia was very similar. The most remarkable difference between them was the number of spines on the hind tibia, which was unstable in this subfamily. Therefore, Gorochov \& Storozhenko (1992) insisted that the number of spines on the hind tibia is completely inappropriate to be used to differentiate genera and transferred the subgenus Gymnaeta to the genus Diestrammena. Moreover, according to the minor differences in the structures of male genitalia, the subgenus Tachycines may also have to be placed in the genus Diestrammena. However, Qin et al. (2018) followed Karny's classification system and supposed that the arrangement of spines on the hind tibia could be used to identify not only the two genera but also other genera in Aemodogryllini. Therefore, they reinstated the status of the genus Tachycines and retransferred the subgenera Gymnaeta and Tachycines from the genus Diestrammena to the genus Tachycines (Qin et al. 2018). At present, species belonging to the subgenus Gymnaeta can easily be identified according to the male genitalia and the absence of spines on the hind femora. The most controversial question is whether the spines on the hind tibia could be used as a generic character to classify Diestrammena and Tachycines. In the absence of sufficient evidence to clarify this issue, no changes have been made to the current classification system and the species are temporarily placed in the genus Tachycines. More evidence is needed for further study.

The South China Karst is one of the world's biodiversity hotspots with an abundant diversity of species (Shu et al. 2013; Monro et al. 2018). Up to now, approximately 145 species of Rhaphidophoridae are recorded from China, among which only 25 species are described from caves.

Cave crickets have developed some characteristics to adapt to cave life, such as a light body color, different degrees of degenerated eyes and fastigium verticis, longer appendages, etc. This fauna represents a good model to study biogeographical issues (Weckstein et al. 2016), and especially to discuss the current distribution pattern of cave species in relation to the geology and the geomorphology, as well as to analyze the relationships between epigean and subterranean species.

However, habitats of cave systems are easily destroyed and cannot be recovered and many undescribed species are in danger of extinction (Jaffé et al. 2016; Borges et al. 2019).

\section{Acknowledgments}

We are grateful to Prof. Mingyi Tian and his team for collecting the specimens, as well as to Mr Wei Yang. We also thank Dr Sigfrid Ingrisch and topical editor Nesrine Akkari for reviewing the manuscript and the second anonymous reviewer who really provided helpful comments that helped the manuscript. The project is supported by the National Natural Science Foundation of China (No. 31872268, 31750002, 31672259) and the Post-graduate's Innovation Fund Project of Hebei Province (CXZZBS2020020).

\section{References}

Adelung N. 1902. Beitrag zur Kenntnis der Paläarctischen Stenopelmatiden (Orthoptera, Locustodea). Annuaire du Musée zoologique de l'Académie impériale des Sciences de St. Pétersbourg 7: 55-75.

Borges P., Lamelas-Lopez L., Amorim I., Danielczak A., Boieiro M., Rego C., Wallon S., Nunes R., Cardoso P. \& Hochkirch A. 2019. Species conservation profiles of cave-dwelling arthropods from Azores, Portugal. Biodiversity Data Journal 7: e32530. https://doi.org/10.3897/BDJ.7.e32530

Cigliano M.M., Braun H., Eades D.C. \& Otte D. 2020. Orthoptera Species File Online. Version 5.0/5.0. Available from http://Orthoptera.SpeciesFile.org [accessed 2 Dec. 2020]. 
Feng X.L., Huang S.H. \& Luo C.Q. 2019. A new species of the subgenus Tachycines (Gymnaeta) (Orthoptera: Rhaphidophoridae) from karst caves of southern Guizhou, China. Zootaxa 4674 (4): $491-$ 495. https://doi.org/10.11646/zootaxa.4674.4.8

Feng X.L., Huang S.H. \& Luo C.Q. 2020a. Three new cave species of the subgenus Tachycines (Gymnaeta) (Orthoptera: Rhaphidophoridae: Aemodogryllinae) from northern Guizhou, China. Zootaxa 4820 (3): 563-571. https://doi.org/10.11646/zootaxa.4820.3.9

Feng X.L., Huang S.H. \& Luo C.Q. 2020b. A new cave species of the genus Gymnaetoides (Rhaphidophoridae: Aemodogryllinae: Aemodogryllini) from Guizhou, China. Zootaxa 4885 (3): $447-$ 450. https://doi.org/10.11646/zootaxa.4885.3.8

Feng Z.G., Wynne J.J. \& Zhang F. 2020. Cave-dwelling pseudoscorpions of China with descriptions of four new hypogean species of Parobisium (Pseudoscorpiones, Neobisiidae) from Guizhou Province. Subterranean Biology 34: 61-98. https://doi.org/10.3897/subtbiol.34.49586

Gao Z.Z., Zhang F. \& Chen H.M. 2020. Two new cave-dwelling species of Tyrannochthonius Chamberlin 1929 (Pseudoscorpiones: Chthoniidae) from the Guizhou karst, China. Zootaxa 4853 (4): 572-580. https://doi.org/10.11646/zootaxa.4853.4.6

Gorochov A.V. \& Storozhenko S.Y. 1992. On the fanua of the subfamily Aemodogryllinae (Orthoptera, Rhaphidophoridae) in Vietnam. Proceedings of the Zoological Institute of the Russian Academy of Science 245: 17-34.

Gorochov A.V., Rampini M. \& Di Russo C. 2006. New species of the genus Diestrammena (Orthoptera: Rhaphidophoridae: Aemodogryllinae) from caves of China. Russian Entomological Journal 15: 355360 .

Jaffé R., Prous X., Zampaulo R., Giannini T.C., Imperatriz-Fonseca V.L., Maurity C., Oliveira G., Brandi I.V. \& Siqueira J.O. 2016. Reconciling mining with the conservation of cave biodiversity: a quantitative baseline to help establish conservation priorities. PLOS ONE 11 (12): e0168348.

https://doi.org/10.1371/journal.pone.0168348

Jiang X.K., Guo X., Chen H.M. \& Xie Z.C. 2018. Four new species of the Glyphiulus javanicus group from southern China (Diplopoda, Spirostreptida, Cambalopsidae). In: Stoev P. \& Edgecombe G.D. (eds) Proceedings of the $17^{\text {th }}$ International Congress of Myriapodology, Krabi, Thailand. Zookeys 741: 155179. https://doi.org/10.3897/zookeys.741.23223

Jiao Z.J., Niu C.Y., Liu X.W., Lei C.L. \& Bi W.X. 2008. Descriptions of Chinese species of the subgenus Diestrammena (Gymnaeta) Adelung (Orthoptera: Rhaphidophoridae). Zootaxa 1917 (1): 55-60. https://doi.org/10.11646/zootaxa.1917.1.4

Karny H.H. 1934. Zur Kenntnis der ostasiatischen Rhaphidophorinen (Orth. Salt. Gryllacrididae). Konowia, Zeitschrift für Systematische Insektenkunde 13 (1-3): 214-230.

Liu W.X. \& Wynne J.J. 2019. Cave millipede diversity with the description of six new species from Guangxi, China. Subterranean Biology 30: 57-94. https://doi.org/10.3897/subtbiol.30.35559

Ma Z.J., Huang S.B. \& Tian M.Y. 2020. Two new species of cavernicolous trechines from central Guizhou and northwestern Guangxi, China (Coleoptera: Carabidae: Trechinae). Zootaxa 4861 (4): 581593. https://doi.org/10.11646/zootaxa.4861.4.6

Monro A.K., Bystriakova N., Fu L., Wen F. \& Wei Y. 2018. Discovery of a diverse cave flora in China. PLoS ONE 13 (2): e0190801. https://doi.org/10.1371/journal.pone.0190801

Qin Y.Y., Wang H.Q., Liu X.W. \& Li K. 2018. Divided the genus Tachycines Adelung (Orthoptera: Rhaphidophoridae: Aemodogryllinae: Aemodogryllini) from China. Zootaxa 4374 (4): 451-475. https://doi.org/10.11646/zootaxa.4374.4.1 
Qin Y.Y., Liu X.W. \& Li K. 2019. Review of the subgenus Tachycines (Gymnaeta) Adelung, 1902 (Orthoptera, Rhaphidophoridae, Aemodogryllinae, Aemodogryllini). Zootaxa 4560 (2): 273-310. https://doi.org/10.11646/zootaxa.4560.2.3

Rampini M., Di Russo C. \& Cobolli M. 2008. The Aemodogryllinae cave crickets from Guizhou, southern China (Orthoptera: Rhaphidophoridae). Monografie naturalistiche 3: 129-141.

Shu S.S., Jiang W.S., Whitten T., Yang J.X. \& Chen X.Y. 2013. Drought and China's cave species. Science 340 (6130): 272. https://doi.org/10.1126/science.340.6130.272-a

Tian M.Y., Huang S.B. \& Wang D.M. 2017. Discovery of a most remarkable cave-specialized trechine beetle from southern China (Coleoptera, Carabidae, Trechinae). ZooKeys 725: 37-47. https://doi.org/10.3897/zookeys.725.21040

Weckstein J.D., Johnson K.P., Johnson J.D., Krejca J.K., Takiya D.M., Veni G., Reddell J.R. \& Taylor S.J. 2016. Comparative phylogeography of two codistributed subgenera of cave crickets (Orthoptera: Rhaphidophoridae: Ceuthophilus spp.). Journal of Biogeography 43 (7): 1450-1463.

https://doi.org/10.1111/jbi.12734

Zhao H.F., Li S.Q. \& Zhang A.B. 2020. Taxonomic revision of Telemidae (Arachnida, Araneae) from East and Southeast Asia. ZooKeys 933: 15-93. https://doi.org/10.3897/zookeys.933.38653

Zhou X.L. \& Yang W.C. 2020. A new species of Tachycines Adelung, 1902 (Orthoptera, Rhaphidophoridae, Aemodogryllinae, Aemodogryllini) from karst caves in Guizhou, China. ZooKeys 937: 21-29. https://doi.org/10.3897/zookeys.937.49173

Zhu Q.D., Chen H.M. \& Shi F.M. 2020. Remarks on the genus Tachycines Adelung, 1902 (Orthoptera: Rhaphidophoridae: Aemodogryllinae) with description of eight new species from caves in southern China. Zootaxa 4809 (1): 71-94. https://doi.org/10.11646/zootaxa.4809.1.4

Manuscript received: 23 December 2020

Manuscript accepted: 21 June 2021

Published on: 19 August 2021

Topic editor: Nesrine Akkari

Desk editor: Kristiaan Hoedemakers

Printed versions of all papers are also deposited in the libraries of the institutes that are members of the EJT consortium: Muséum national d'histoire naturelle, Paris, France; Meise Botanic Garden, Belgium; Royal Museum for Central Africa, Tervuren, Belgium; Royal Belgian Institute of Natural Sciences, Brussels, Belgium; Natural History Museum of Denmark, Copenhagen, Denmark; Naturalis Biodiversity Center, Leiden, the Netherlands; Museo Nacional de Ciencias Naturales-CSIC, Madrid, Spain; Real Jardín Botánico de Madrid CSIC, Spain; Zoological Research Museum Alexander Koenig, Bonn, Germany; National Museum, Prague, Czech Republic. 